\section{Nervengift oder Neuromodulation bei überaktiver Blase?}

Wenn konservative Therapien der überaktiven Blase versagen, kommen
minimal-invasive Behandlungsstrategien zum Zuge. In einer aktuellen
Studie wurde nun anhand einer Kosten-Nutzen-Analyse geprüft, ob sich
Botulinumtoxin-Injektionen oder die sakrale Neuromodulation besser eignet.

$\mathrm{n}$ einer Studie wurde anhand des Ko-

sten-Nutzen-Verhältnisses ermittelt, ob die Injektion von Botulinumtoxin A (BTX) in den Detrusor oder die sakrale Neuromodulation (SNM) die geeignetere Methode zur Behandlung der therapierefraktären überaktiven Blase (OAB) ist. Grundlage der Kosten-Nutzen-Analyse waren Studien und, wenn notwendig, Expertenmeinungen zur Wirksamkeit von BTX und SNM sowie zum Krankheitsverlauf unter diesen Therapien. Vorausgesetzt wurde, dass die Injektion oder Implantation unter Vollnarkose erfolgen und das Ansprechen auf SNM mit ,tinedlead“-Elektroden getestet wird. Ein klinisch relevanter Nutzen war definiert als
Rückgang der Inkontinenzepisoden oder des imperativen Harndrangs und der Miktionsfrequenz um mindestens 50\%. Bei den Kosten wurden nicht nur die direkten Therapiekosten, sondern auch alle weiteren Kosten wie durch Nebenwirkungen oder bei Therapieversagen berücksichtigt.

Für einen Behandlungszeitraum von fünf Jahren wurde für die beiden Verfahren eine vergleichbar hohe Erfolgsquote von etwa $75 \%$ errechnet. Wegen des Wirkungsverlustes zwischen den BTX-Injektionen werden mit dieser Therapie aber nur 4,72 qualitätsadjustierte Lebensjahre (QALY) erreicht gegenüber 4,95 mit SNM. Die bessere Wirkung der SNM $(0,23$
QALY pro Patient) ist nach dieser Modellrechnung mit zusätzlichen Kosten von 6.428 EUR verbunden (25.780 EUR vs. 19.353 EUR mit BTX). Die Mehrkosten für ein QALY betragen damit 27.991 EUR. Dieser Wert liegt nach Aussage der Studienautoren unterhalb des in Industrienationen üblichen Grenzwertes.

Fazit: Die Autoren beurteilen den Beginn einer Behandlung mit SNM als kosteneffektiver im Vergleich zum Start mit BTX. Allerdings wird die Kosteneffektivität der SNM erst nach vier Jahren erreicht. Darüber hinaus ist sie auch nach fünf Jahren noch nicht gegeben, falls die BTX-Injektion in Lokalanästhesie erfolgt oder für die SNM ein PNE- oder bilateraler Test durchgeführt wird. Dr. Beate Schumacher

Leong RK et al. Cost-effectiveness analysis of sacral neuromodulation and botulinumtoxin A treatment for patients with idiopathic overactive bladder. BJU Int 2011; 108: 558-64

\title{
Knötchen in der Skrotalhaut
}

\section{Manche Erkrankungen bleiben jahrelang im Verborgenen und werden oft erst Jahre später als Zufallsbefund entdeckt. Das verdeutlicht das Beispiel einer Calcinosis scrotalis, die den betroffenen Patienten nicht zu stören schien.}

$E_{h}^{\mathrm{i}}$ in 32-jähriger Mann suchte die Nothilfe wegen Dyspnoe und Hämoptysen auf. Bei der körperlichen Untersuchung wurden als Zufallsbefund weißlich-gelbe Knötchen in der Skrotalhaut fest gestellt (Abbildung links). Auf Nachfragen berichtete der Mann, dass sich dieser Befund innerhalb der letzten zehn bis zwölf Jahre entwickelt habe. Die in-

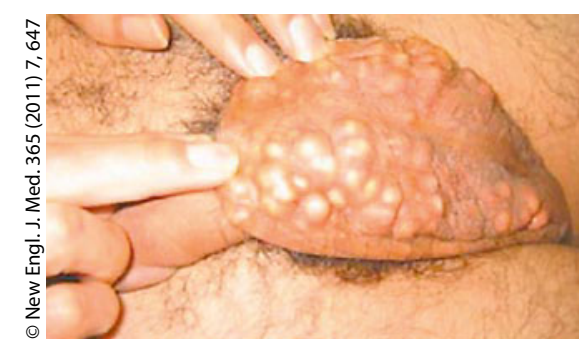

dolenten derben Knötchen erlaubten die klinische Blickdiagnose einer Calcinosis scrotalis.

Allerdings war das nicht das einzige medizinische Problem des Mannes. In der Kontrastmittel-verstärkten Computertomografie (CT) des Thorax und des Abdomens fanden sich zahlreiche kleine Lungenemboli, worauf man eine Antiko-

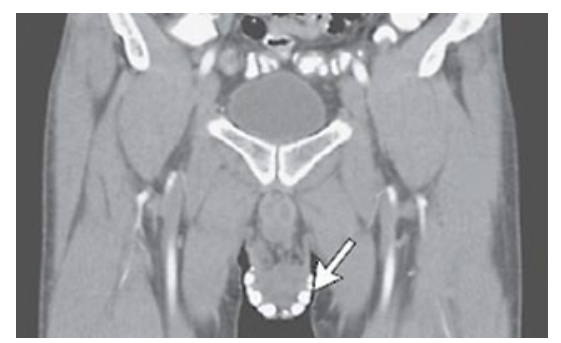

Calcinosis scrotalis: klinisches Bild (links) und Computertomogramm (rechts) agulation begann. In der CT des Beckens stellten sich die intrakutan liegenden verkalkten Knoten der Skrotalhaut in einer Größe von 3-8 mm dar (Abbildung rechts, Pfeil).

Bei Calcinosis scrotalis handelt es sich um eine gutartige Veränderung ungeklärter Ätiopathogenese, die in der Regel in der Pubertät und im früheren Erwachsenenalter beginnt. Störungen des Kalzium- oder Phosphatsstoffwechsels liegen nicht vor. Die intradermal gelegenen Knoten nehmen meist im Laufe von Jahren an Größe zu und können exulzerieren, sodass sich weißliches, kreideartiges Material entleert. Die meisten Patienten wollen die Knoten aus ästhetischen Gründen entfernt haben. Dieser Patient schien sich wenig daran zu stören, ließ die Verkalkungen in Ruhe und war nur durch seine zahlreichen Lungenemboli beunruhigt. Prof. Hermann S. FüeßI

Grenader T et al. Scrotal calcinosis. New Engl J Med 2011; 365:, 647 